\title{
Effects of loading contact on electric-power generation of lead zirconate titanate piezoelectric ceramic plate
}

\author{
Mitsuhiro OKAYASU*, Tsukasa OGAWA \\ Graduate School of Natural Science and Technology, Okayama University, Okayama 700-8530, Japan
}

Received: December 20, 2018; Revised: April 10, 2019; Accepted: April 15, 2019

(C) The Author(s) 2019.

\begin{abstract}
To better understand the generation of electric power for piezoelectric $\mathrm{PbZrTiO}_{3}(\mathrm{PZT})$ ceramic plate $(\phi 25 \mathrm{~mm})$, an attempt was made to investigate experimentally and numerically electricpower generation characteristics during cyclic bending under various loading fixtures $(\phi 0-\phi 20 \mathrm{~mm})$, i.e., different contact areas. Increasing the load-contact area on the PZT ceramic leads to a nonlinear decrease in the generated voltage. Decreasing contact area basically enhances the generated voltage, although the voltage saturates during loading when the contact area is less than $\phi 5 \mathrm{~mm}$. A similar voltage is generated for $\phi 0$ and $\phi 5 \mathrm{~mm}$, which is attributed to strain status (ratio of compressive and tensile strain) and material failure due to different stress distribution in the PZT ceramic. On the basis of the obtained electric generation voltage, suitable loading conditions are clarified by loading with the $\phi 5 \mathrm{~mm}$ fixture, which generates a higher voltage and a longer lifetime of the PZT ceramic. From this approach, it is appeared that the area contact with the area ratio of $0.04(\phi 5 \mathrm{~mm} / \phi 20 \mathrm{~mm})$ is suitable to obtain the high efficiency of the electric voltage.
\end{abstract}

Keywords: piezoelectric ceramic; lead zirconate titanate ceramic; electric power generation

\section{Introduction}

To reduce demands on the environment, clean energy systems are urgently required in modern society. In response, various clean-energy systems have been developed in recent years, such as wind energy, solar energy, wave energy, etc. Another approach involves the use of smart materials to make energy-harvesting systems, which has received intense attention due to their limitless possibilities. The emphasis in such cases has been to develop on-site generators that can transform any available electrical energy [1]. Piezoelectric

\footnotetext{
* Corresponding author.

E-mail: mitsuhiro.okayasu@utoronto.ca
}

energy harvesting has emerged as one of the prime methods for transforming mechanical energy into electric energy. The harvesting devices consist of disk-shaped piezoelectric ceramics set in a checkerboard pattern. Piezoelectric ceramics can also be stored and used to power devices in remote situations [2]. To develop piezoelectric energy-harvesting systems, many researchers have investigated electric generation in $\mathrm{PbZrTiO}_{3}(\mathrm{PZT})$ ceramics. In their review articles, Cook-Chennault et al. [3] discuss fundamental systems and future trends and applications for piezoelectric energy harvesting. Sodano et al. [4] have developed a technique to generate electricity from the vibration of a piezoelectric material, which they then use to recharge a discharged nickelmetal-hydride battery. Tadesse et al. [5] proposed two different mechanisms, piezoelectric and electromagnetic, 
that they combined them on a single platform for energy harvesting.

Energy harvesting with a PZT ceramic is not very efficient, so an optimized harvesting technique is required. Several investigators have analyzed numerically and experimentally the efficiency of electric-power generation with a PZT [6]. For example, one of the present authors investigated electric-power generation in a PZT ceramic during cyclic loading, in which the maximum voltage generated depends on the frequency and the wave shape (e.g., triangular or sinusoidal wave mode). The maximum voltage for both wave modes increases with increasing frequency and saturates around $15 \mathrm{~Hz}$, at which the mean voltage and current are about $8 \mathrm{~V}$ and $0.02 \mathrm{~mA}$, respectively [7]. Electric-power generation was examined under cyclic loading and with different loading conditions [8]; however, the role played by strain in PZT ceramics has yet to be analyzed systematically and in detail. Because strain in PZT ceramics is one of the main factors that determine the properties of electric-power generation in these materials, the loading conditions must be designed very carefully. In the present work, to minimize stress concentration, the mechanical loading was considered with a wide area contact to reduce the material failure, although this approach may decrease the efficiency of electric-power generation. Thus, an attempt was made to propose an
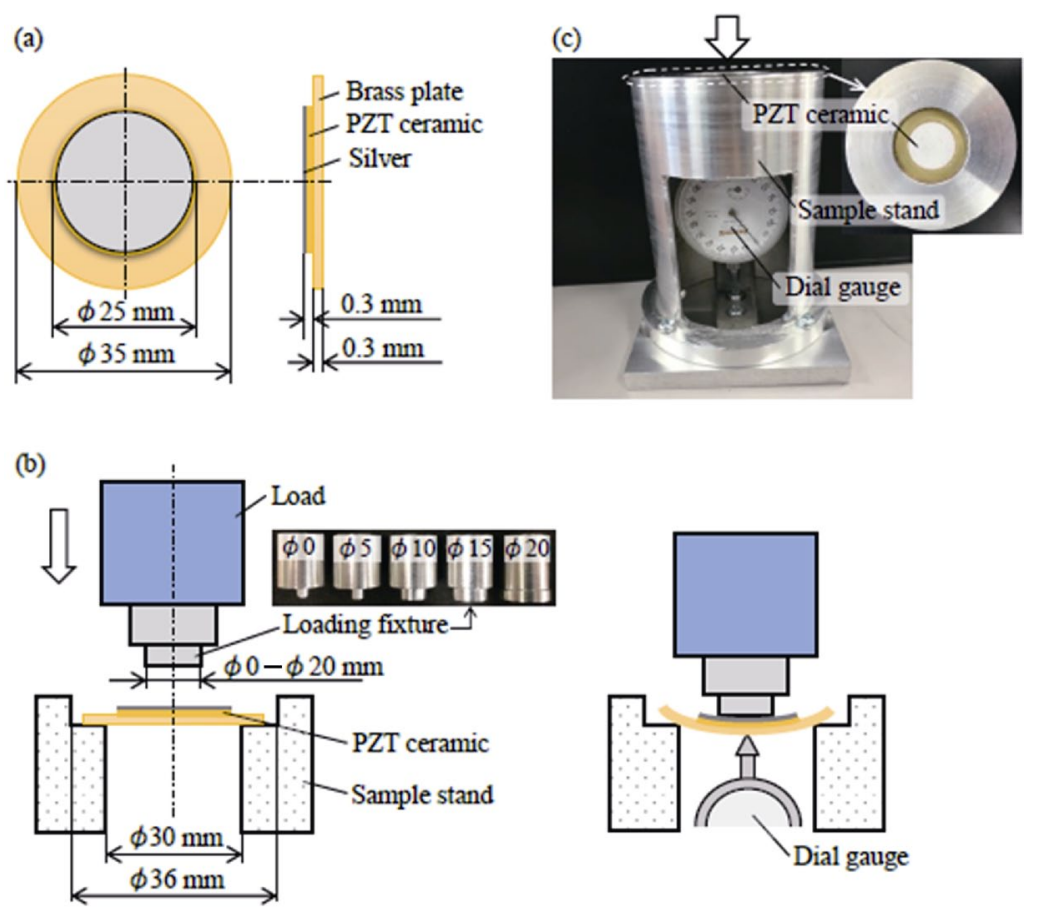

Fig. 1 Schematic diagrams of (a) PZT ceramic plate, (b) loading apparatus for investigating electric-power generation by PZT ceramic plate, and (c) apparatus to measure displacement. 
diagram of the test apparatus with the PZT ceramic plate and the loading fixture. The PZT ceramic plate was placed on the sample stand to create a bending load. The loading fixtures allowed us to do three or four point-like bending tests. The loading fixtures and sample stand were made of aluminum alloy to give them high stiffness compared with the PZT ceramic. The PZT ceramic plate was cyclically loaded in air by using a screw-driven universal testing machine with $50 \mathrm{kN}$ capacity (Shimazu, AG-Xplus). In this case, cyclic loading was done by a square-wave loading mode with $R$-ratio $\left(\sigma_{\min } / \sigma_{\max }\right)=0.05$ and frequency $f=0.25 \mathrm{~Hz}$. To investigate how the loading condition affects electric-power generation, the PZT ceramic was loaded by round rods of varying diameters $(\phi 0, \phi 5, \phi 10, \phi 15$, and $\phi 20 \mathrm{~mm})$. Note that loading with the $\phi 0 \mathrm{~mm}$ fixture corresponds to a point contact. The deflection and strain of the PZT ceramic plate during cyclic loading was directly measured using a dial gauge (Figs. 1(b) and 1(c)) and a commercial strain gauge, which were fixed to the brass plate. The strain gauge was fixed at three different points: 0,5 , and $10 \mathrm{~mm}$ from the center of the PZT ceramic.

During the cyclic loading, the electric power generated from the PZT ceramic was monitored by using a digital multimeter (8846A, Fluke). In this approach, the mean maximum voltage was employed as a parameter to quantitatively evaluate the electric-power generation characteristics. For this evaluation, cyclic loading was applied at $10-50 \mathrm{~N}$ for $30 \mathrm{~s}$. Figure 2 shows the experimentally obtained voltage versus time upon cyclical loading to the PZT ceramic. The results show that the positive electric voltage rises sharply to a maximum level (+) as the bending load is applied to the PZT ceramic. In this time range, the electric-power generation causes electrons to flow in the PZT ceramic. As indicated by the dashed arrow $(※)$, the electric

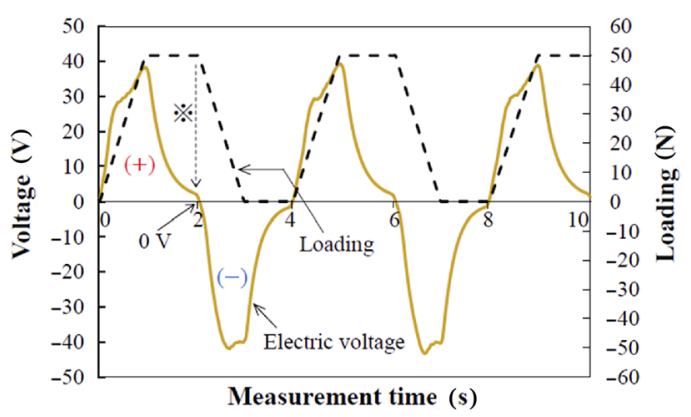

Fig. 2 Voltage generated by PZT ceramic and mechanical loading of PZT ceramic as a function of time. voltage drops to $0 \mathrm{~V}$ despite a constant, nonzero bending load; at $0 \mathrm{~V}$, the electrons that flow out of the material as the load increases have now flowed back into the material [8]. Decreasing the applied load generates a negative electric voltage $(-)$, so the current in the PZT ceramic reverses.

\section{2 Finite-element (FE) analysis}

To analyze numerically strain characteristics and electric-power generation of the PZT ceramic, a FE analysis was carried out using commercial software. Figure 3 shows a schematic diagram of the test fixture and test samples, which consist of a round PZT ceramic and brass plates, which were designed to fit the geometry of the test apparatus with the PZT ceramic plate (Fig. 1). The two-dimensional FE simulations with eight-node quad elements were conducted using ANSYS 15.0. For the ceramic plate, a mesh size of less than $0.01 \mathrm{~mm}$ was used. The material properties employed in this analysis are as follows: an elastic constant $E=$ $82 \mathrm{GPa}$ and a Poisson ratio $v=0.30$ for the PZT ceramic, and $E=103 \mathrm{GPa}$ and $v=0.35$ for the brass plate. In this case, the loading fixture and sample stand were taken to be rigid structures.

In addition, with commercial software (MARC 2008), the electric voltage of the PZT ceramic under a bending load was estimated. The electric displacement $D$ (i.e., electric energy) can be calculated by

$$
D=T d+\varepsilon^{T} e
$$

where $T$ is the mechanically applied stress, $d$ is the piezoelectric constant, $\varepsilon^{T}$ is the permittivity, and $e$ is the electric field. Since the electric field is very small during the mechanical loading, the term $\varepsilon^{T} e$ may be omitted, which gives:

$$
D=T d
$$

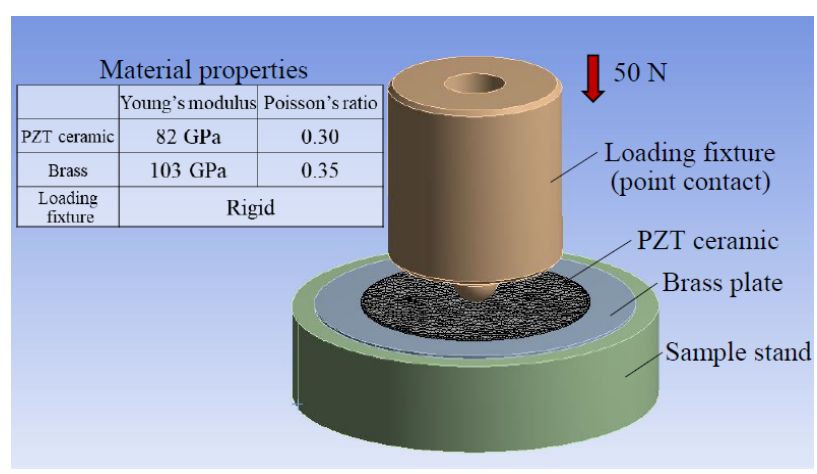

Fig. 3 FE model with material parameters for simulating strain characteristic and electric field in PZT ceramic. 


$$
\begin{aligned}
{\left[\begin{array}{l}
D_{1} \\
D_{2} \\
D_{3}
\end{array}\right]=} & {\left[\begin{array}{llllll}
d_{11} & d_{12} & d_{13} & d_{14} & d_{15} & d_{16} \\
d_{21} & d_{22} & d_{23} & d_{24} & d_{25} & d_{26} \\
d_{31} & d_{32} & d_{33} & d_{34} & d_{35} & d_{36}
\end{array}\right]\left[\begin{array}{l}
T_{1} \\
T_{2} \\
T_{3} \\
T_{4} \\
T_{5} \\
T_{6}
\end{array}\right] } \\
& +\left[\begin{array}{lll}
\varepsilon_{11} & \varepsilon_{12} & \varepsilon_{13} \\
\varepsilon_{12} & \varepsilon_{22} & \varepsilon_{23} \\
\varepsilon_{13} & \varepsilon_{23} & \varepsilon_{33}
\end{array}\right]\left[\begin{array}{l}
E_{1} \\
E_{2} \\
E_{3}
\end{array}\right]
\end{aligned}
$$

The electric charge $q$ in the PZT ceramic is

$$
q=A D
$$

where $A$ is the surface area of the PZT ceramic. By combining Eqs. (2) and (3), the electric charge $q$ takes the form:

$$
q=\left[A_{1} A_{2} A_{3}\right]\left[\begin{array}{llllll}
d_{11} & d_{12} & d_{13} & d_{14} & d_{15} & d_{16} \\
d_{21} & d_{22} & d_{23} & d_{24} & d_{25} & d_{26} \\
d_{31} & d_{32} & d_{33} & d_{34} & d_{35} & d_{36}
\end{array}\right]\left[\begin{array}{c}
T_{1} \\
T_{2} \\
T_{3} \\
T_{4} \\
T_{5} \\
T_{6}
\end{array}\right]
$$

where $F$ is the applied force. The electric charge is also assessed by the voltage $V$ and braking capacitance $C_{d}$ [9]:

$$
V=q / C_{d}
$$

In this case, $C_{d}$ is associated with the dielectric constant $\varepsilon$ and the geometry of the PZT ceramic:

$$
C_{d}=A \varepsilon / l
$$

$\left[\begin{array}{lll}C_{1} & C_{2} & C_{3}\end{array}\right]=\left[\begin{array}{lll}A_{1} & A_{2} & A_{3}\end{array}\right]\left[\begin{array}{lll}\varepsilon_{11} & \varepsilon_{12} & \varepsilon_{13} \\ \varepsilon_{12} & \varepsilon_{22} & \varepsilon_{23} \\ \varepsilon_{13} & \varepsilon_{23} & \varepsilon_{33}\end{array}\right] / l$

where $l$ is the length of the PZT ceramic. Substituting Eqs. (6) and (4) into Eq. (5) leads to

$$
V=d T l / \varepsilon
$$

$$
\begin{aligned}
{\left[\begin{array}{l}
V_{1} \\
V_{2} \\
V_{3}
\end{array}\right]=} & {\left[\begin{array}{llllll}
d_{11} & d_{12} & d_{13} & d_{14} & d_{15} & d_{16} \\
d_{21} & d_{22} & d_{23} & d_{24} & d_{25} & d_{26} \\
d_{31} & d_{32} & d_{33} & d_{34} & d_{35} & d_{36}
\end{array}\right]\left[\begin{array}{l}
T_{1} \\
T_{2} \\
T_{3} \\
T_{4} \\
T_{5} \\
T_{6}
\end{array}\right] l } \\
& /\left[\begin{array}{lll}
\varepsilon_{11} & \varepsilon_{12} & \varepsilon_{13} \\
\varepsilon_{12} & \varepsilon_{22} & \varepsilon_{23} \\
\varepsilon_{13} & \varepsilon_{23} & \varepsilon_{33}
\end{array}\right]
\end{aligned}
$$

\section{Results and discussion}

\section{1 Characteristics of electric-power generation}

To understand the electric generation characteristics of the PZT ceramic, the electric voltage was examined experimentally in advance. Figure 4 shows the mean maximum voltage as a function of the maximum cyclic loading value. The voltage clearly increases with increasing applied loading, although the rate of increase depends on the size of the loading fixture: a larger contact area corresponds to a smaller voltage. The voltage for the $\phi 20 \mathrm{~mm}$ fixture is as low as $45 \mathrm{~V}$ at $50 \mathrm{~N}$, which is about $60 \%$ of that for the $\phi 0$ and $\phi 5 \mathrm{~mm}$ fixtures. Note that the data for the voltage generated by the $\phi 0 \mathrm{~mm}$ fixture are superposed on those for the $\phi 5 \mathrm{~mm}$ fixture. To understand clearly how contact area affects electric-power generation, the data are summarized in Fig. 5, which shows the voltage generated for each loading fixture. Note that the percentages indicated in Fig. 5 give the voltage with respect to that obtained with the $0 \%$ fixture. A higher voltage is generated for the smaller contact areas (i.e., $\phi 0$ and $\phi 5 \mathrm{~mm}$ fixtures), which is attributed to the high stress (or high strain) generated in the PZT ceramic in these cases. This trend also occurs at different loading values. However, this result is questionable because, for a given load, the bending stress (or strain) for the $\phi 5 \mathrm{~mm}$ fixture is less than that for the $\phi 0 \mathrm{~mm}$ fixture. This result may be attributed to various mechanisms, such as the strain characteristics and localized failure in the PZT ceramic. Figure 6 shows photographs of the surface of the PZT ceramic after cyclic loading at $50 \mathrm{~N}$ for $30 \mathrm{~s}$ using the $\phi 0$ and $\phi 5 \mathrm{~mm}$ fixtures. The point contact $(\phi 0 \mathrm{~mm})$ makes a severe dent in the PZT ceramic, whereas no

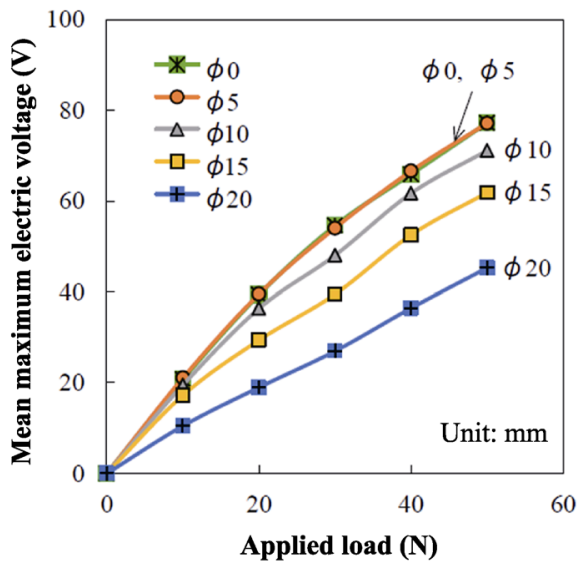

Fig. 4 Mean maximum voltage as a function of load applied by various loading fixtures. 

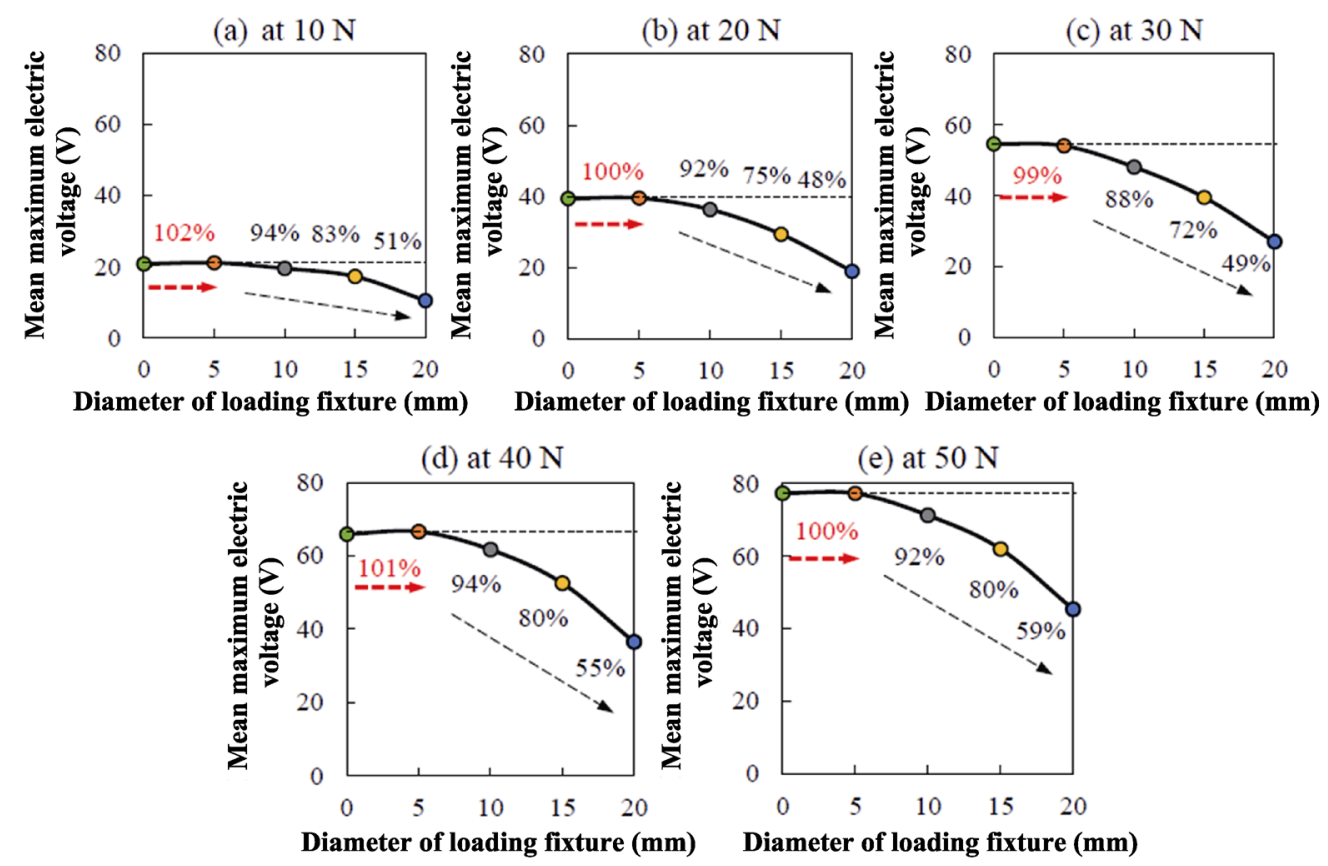

Fig. 5 Mean maximum voltage as a function of loading fixture diameter for various loading values.
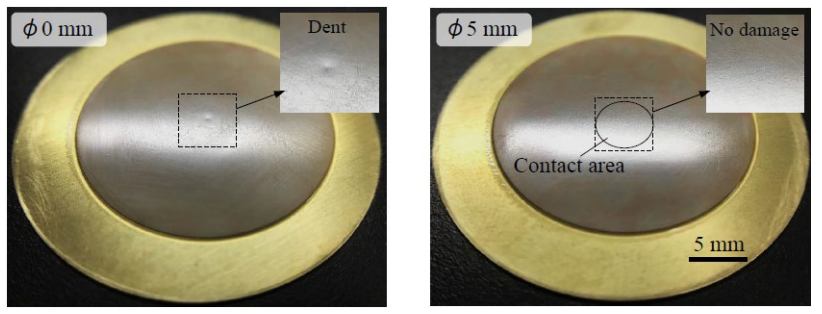

Fig. 6 Photographs of surface of PZT ceramic after cyclic loading with $\phi 0$ and $\phi 5 \mathrm{~mm}$ fixtures, showing localized failure caused by mechanical contact with loading fixture.

clear damage appears on the surface of the PZT ceramic after loading with the $\phi 5 \mathrm{~mm}$ fixture. The dent in the PZT ceramic is the result of localized failure (e.g., domain switching and micro cracks), which results in poor piezoelectric properties.

To clearly understand the characteristics of electricpower generation, the strain in the PZT ceramic plate on both macro and micro scales was investigated experimentally. Figure 7(a) shows the deflection of the PZT ceramic plate as a function of applied load for the different fixtures. The overall deflection increases sublinearly with increasing the loading value. Based on the experimental result shown in Fig. 7(a), deflection value as a function of loading-fixture diameter is indicated in Fig. 7(b), which was obtained under the cyclic loading with the maximum load of $50 \mathrm{~N}$. High deflection occurs for loading fixtures with smaller
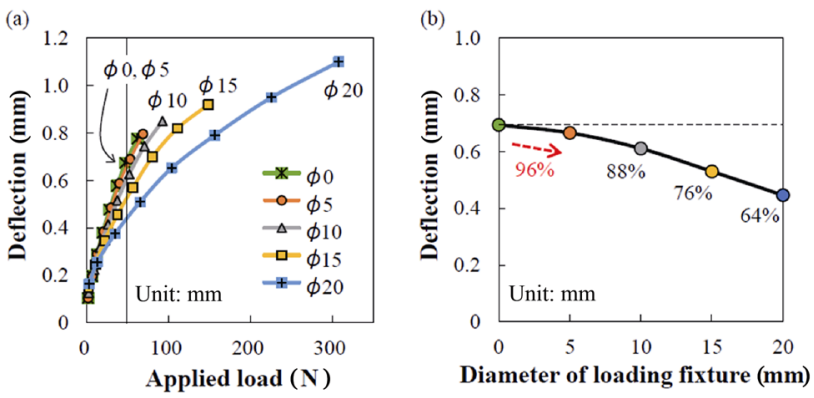

Fig. 7 Deflection of PZT ceramic plate as a function of (a) applied load and (b) loading fixture diameter for $50 \mathrm{~N}$ applied load.

contact areas, and deflection decreases with increasing size of the loading fixture. Although the voltage generated is similar for loading with $\phi 0$ and $\phi 5 \mathrm{~mm}$ fixtures, the $\phi 5 \mathrm{~mm}$ fixture leads to less deflection than the $\phi 0 \mathrm{~mm}$ fixture, e.g., $96 \%$ as indicated in Fig. 7(b).

Using the strain gauges, the strain characteristics of the PZT ceramic were examined. Figure 8 shows the strain obtained at 0,5 , and $10 \mathrm{~mm}$ from the center of the PZT ceramic. At $0 \mathrm{~mm}$, a high strain is obtained when loaded by the point contact $(\phi 0 \mathrm{~mm})$, and the strain decreases with increasing size of the loading fixture (Fig. 8(a)). In contrast, a different trend occurs at 5 and $10 \mathrm{~mm}$ from the center of the PZT ceramic. At $5 \mathrm{~mm}$, the strain is slightly higher when loaded with the $\phi 10 \mathrm{~mm}$ fixture (Fig. 8(b)) than with the other fixtures. This may be due to the edge of the $\phi 10 \mathrm{~mm}$ 
(a) Center position
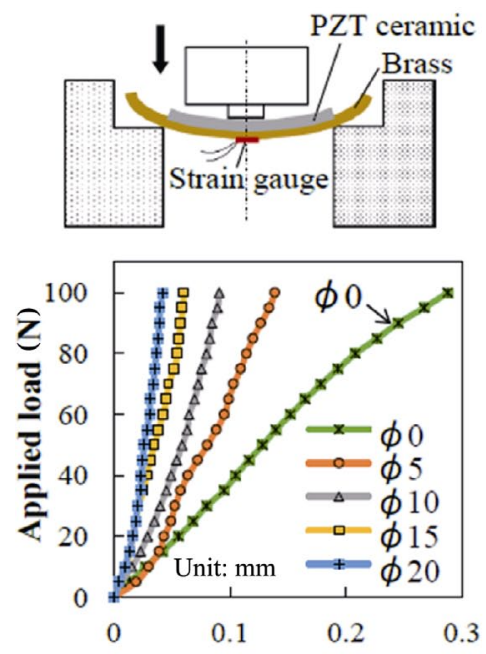

Strain at center point $(\%)$ (b) at $5 \mathrm{~mm}$ from the center
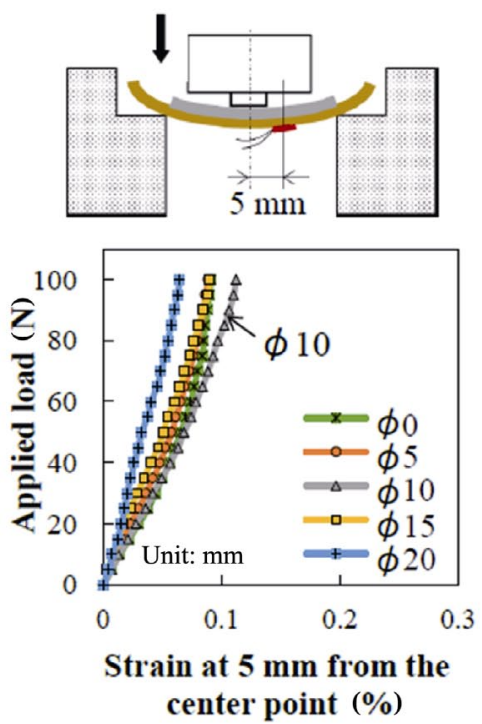

(c) at $10 \mathrm{~mm}$ from the center
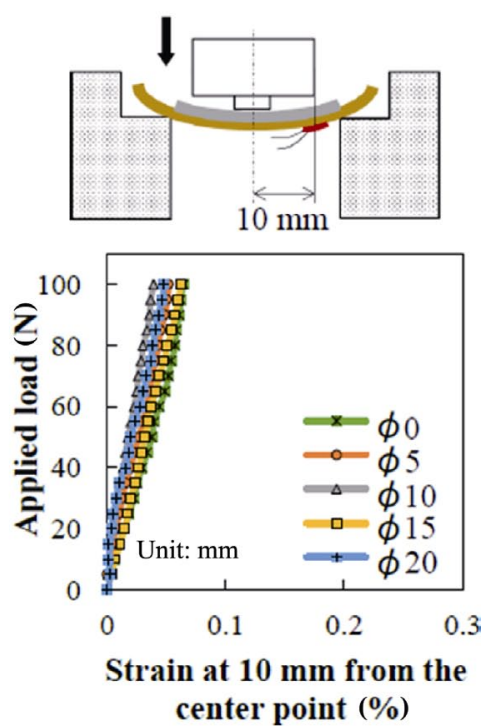

Fig. 8 Applied load as a function of strain at (a) 0, (b) 5, and (c) $10 \mathrm{~mm}$ from the center of PZT ceramic.

loading fixture being located at the position of the strain gauge $(5 \mathrm{~mm})$. Conversely, a similar and small strain is obtained at $10 \mathrm{~mm}$ from the PZT center for all loading fixtures (Fig. 8(c)). This result may be attributed to the high stiffness in the PZT ceramic near the edge of the sample stand and which is due to stacking both the sample stand and the PZT ceramic under the loading process.

\section{2 FE analysis of electric-power generation}

To understand the strain characteristics of the PZT ceramic in detail, a FE analysis was executed. Figure 9 shows an equivalent strain distribution on the surface of a cross section of the PZT ceramic, as calculated by using $\left\{0.5\left[\left(\sigma_{x}-\sigma_{y}\right)^{2}+\left(\sigma_{x}-\sigma_{z}\right)^{2}+\left(\sigma_{z}-\sigma_{x}\right)^{2}\right] / E\right\}^{0.5}$. The extent of the equivalent strain differs, e.g., a smaller contact area leads to a higher generated voltage. Note that almost all areas are dominated by compressive strain, as shown in the axial direction $\left(\sigma_{x} / E\right)$, but a tensile axial strain is also created in the outer area of the PZT ceramic, as indicated by the dashed circle. In this case, the strain level is not uniformly distributed as the PZT ceramic is loaded with the edge of the loading fixture. The region of tensile strain is wider in the PZT ceramic loaded with a small contact area $(\phi 0 \mathrm{~mm})$. Since tensile strain generates a negative voltage [10] (Fig. 10), the generated voltage could have been reduced (Figs. 4 and 5), when the PZT ceramic is loaded with a small loading fixture.

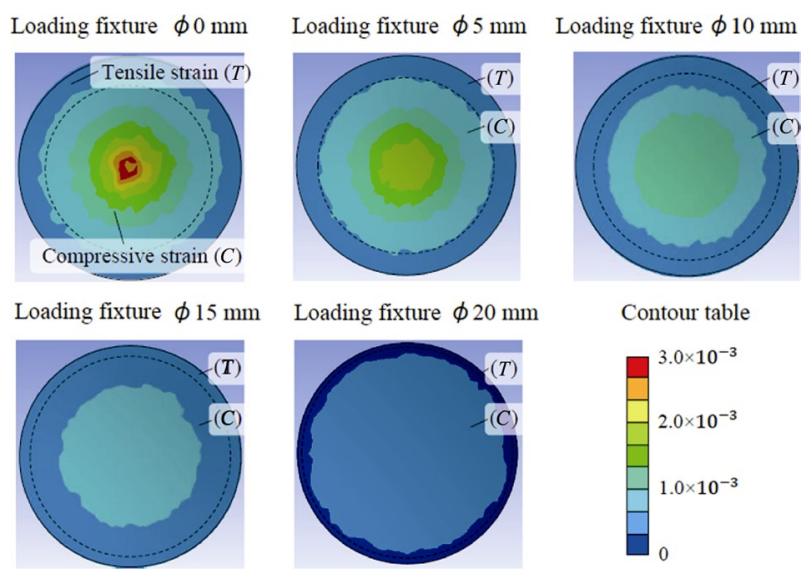

Fig. 9 Distribution of FE strain over PZT ceramic, showing tensile and compressive strains.

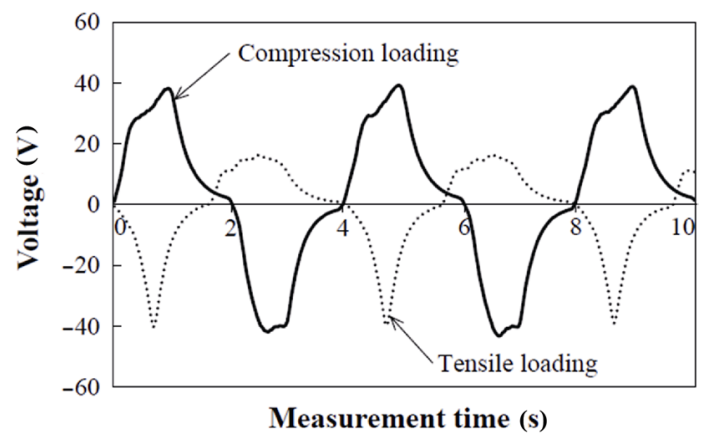

Fig. 10 Voltage generated from PZT ceramic under tensile and compressive stress as a function of measurement time.

Numerical analysis was further conducted to estimate the electric generation characteristics. Figure 11 shows 
Electric field intensity

Loading fixture $\phi 0 \mathrm{~mm}$ (point contact) $\quad \phi 5 \mathrm{~mm}$ (area contact)

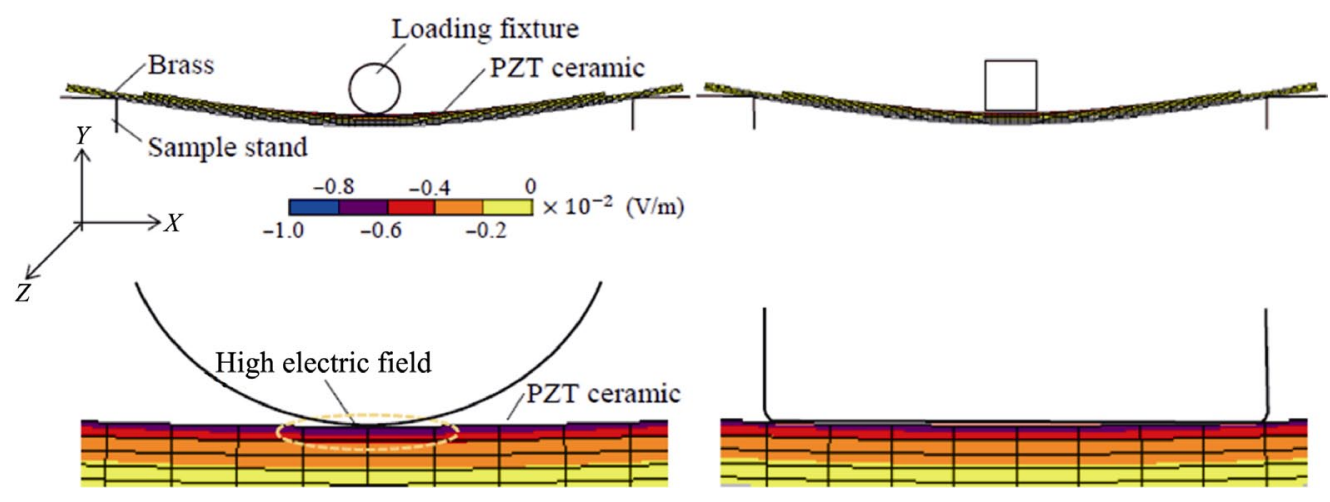

Fig. 11 FE simulation of electric field in the PZT ceramic for loading with $\phi 0$ and $\phi 5 \mathrm{~mm}$ fixtures.

the electric-field intensity on a cross section of the PZT ceramic plate for both the $\phi 0$ and $\phi 5 \mathrm{~mm}$ fixtures. A relatively large electric field appears in the PZT ceramic near the loading point of the $\phi 0 \mathrm{~mm}$ fixture compared with the results for the $\phi 5 \mathrm{~mm}$ fixture. This result is attributed to the high strain in the PZT ceramic caused by $\phi 0 \mathrm{~mm}$ loading. Thus, high electric-power generation may be obtained for $\phi 0 \mathrm{~mm}$ loading, which differs from the trend of the experimental results shown in Fig. 5.

Figure 12 shows the voltage as a function of deflection and bending strain, respectively. Both cases show almostlinear correlations, and particularly high correlation occurs between deflection and electric voltage. From these results, the voltage can be approximated from the strain characteristics even though some data (e.g., $\phi 0 \mathrm{~mm}$ fixture) are slightly shifted to the right-hand side. This may be due to the material damage arising from the point contact, ratio of tensile, and compressive strain as mentioned above, which reduce the voltage. Based on these experimental results, loading with the $\phi 5 \mathrm{~mm}$ fixture is considered to be optimal for generating a high voltage with minimal material damage. It should be

(a) Voltage vs. deflection

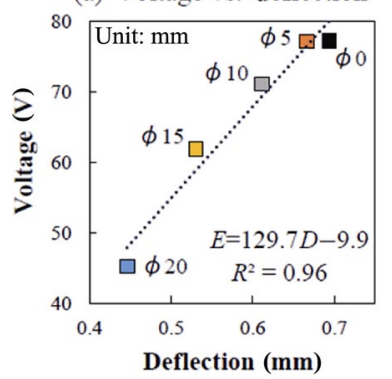

(b) Voltage vs. bending strain

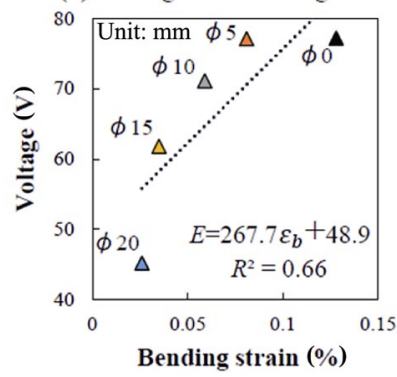

Fig. 12 Voltage generated by PZT ceramic as a function of (a) deflection and (b) bending strain. pointed out that, in this case, the loading fixture with $\phi 5 \mathrm{~mm}$ made the high voltage as the PZT ceramic plate with $\phi 25 \mathrm{~mm}$ is employed. Namely, the area ratio of $0.04(\phi 5 \mathrm{~mm} / \phi 25 \mathrm{~mm})$ is proper contact area to obtain the high efficiency of the electric voltage.

To verify this result, the electric generation characteristics of the PZT ceramic plate were investigated during cyclic loading.

\section{3 Fatigue properties}

Figure 13 shows the voltage as a function of loading-cycle number up to 10,000 cycles for the PZT ceramic with loading fixtures $\phi 0, \phi 5$, and $\phi 15 \mathrm{~mm}$. Two maximum cyclic loads were applied in this approach, 40 and $50 \mathrm{~N}$. In the early fatigue stage ( $<1000$ cycles $)$, the voltage decreases by about $6 \%$ under all conditions. This reduction in voltage is attributed to (i) material damage (e.g., domain switching and generation of micro cracks) [11] and (ii) insufficient current for electric-power generation during cyclic loading (i.e., electrons cannot be completely recovered because of the repeated quick loadings). Note that, at the first $50 \mathrm{~N}$ cycle, the voltage drops rapidly to a low level when loading with the $\phi 0 \mathrm{~mm}$ fixture, as indicated by the arrow with the star, where severe material damage could occur. In addition, after more than 1000 loading cycles, the voltage decreases further with increasing cycle number (with $\phi 0 \mathrm{~mm}$ fixture). This reduction in voltage after more than 1000 cycles (with $\phi 0 \mathrm{~mm}$ fixture) may be attributed to the failure of the PZT ceramic. In the previous work, the material failure (domain switching) of the PZT ceramic was examined by electron back-scattered diffraction (EBSD) analysis before and after the cyclic loading. It 
at $40 \mathrm{~N}$

(a) Loading fixture $\phi 0 \mathrm{~mm}$

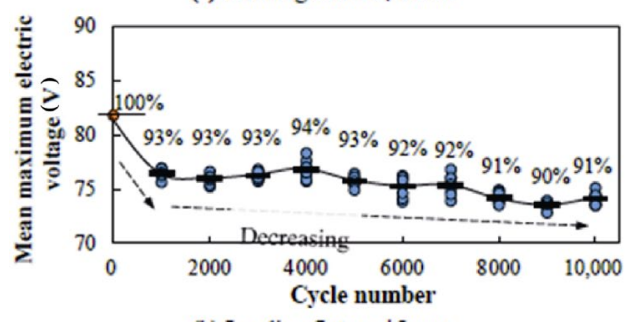

(b) Loading fixture $\phi 5 \mathrm{~mm}$

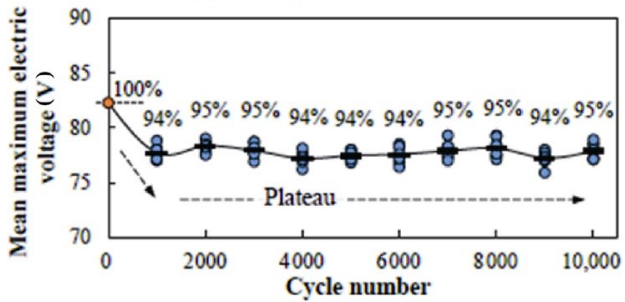

(c) Loading fixture $\phi 15 \mathrm{~mm}$

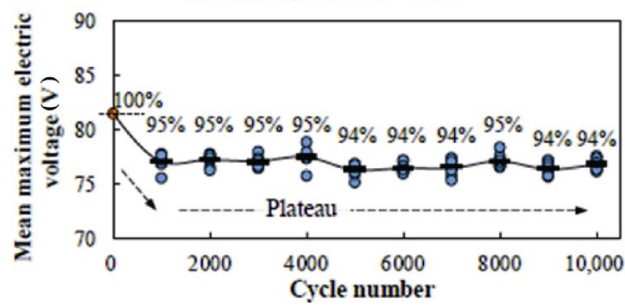

at $50 \mathrm{~N}$

(a)' $\phi 0 \mathrm{~mm}$

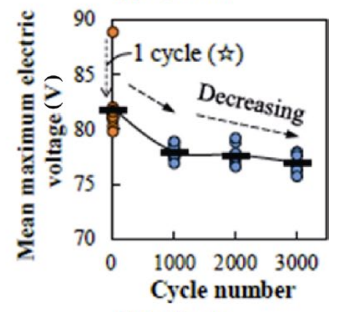

(b)' $\phi 5 \mathrm{~mm}$

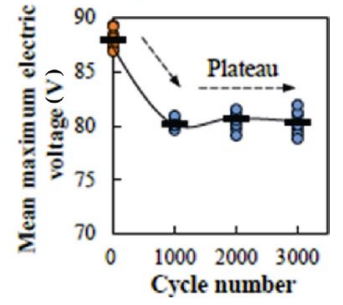

Fig. 13 Maximum voltage generated by PZT ceramic as a function of cycle number for (a) $\phi 0$, (b) $\phi 5$, and (c) $\phi 15$ mm loading fixtures at loads of $40 \mathrm{~N}$ (left column) and $50 \mathrm{~N}$ (right column).

appears that the $90^{\circ}$ domain switching results in a reduction of the electric generation characteristics [11]. Conversely, the voltage generated by loading with the $\phi 5$ and $\phi 10 \mathrm{~mm}$ fixtures becomes relatively stable after 1000 loading cycles. The stability of the voltage after 1000 cycles when loading with the $\phi 5 \mathrm{~mm}$ fixture supports the conclusion that no severe failure occurs in the PZT ceramic. Because of unclear difference in the results of electric generation voltage for the $\phi 0$ and $\phi 5$ $\mathrm{mm}$ fixture, the high cyclic loading of $100 \mathrm{~N}$ was further applied using the loading fixtures $(\phi 0$ and $\phi 5$ $\mathrm{mm})$. The obtained results are shown in Fig. 14. Unlike the results at the low applied loads of 40 and $50 \mathrm{~N}$, significant reduction of the electric voltage during thecyclic loading is appeared for the sample with the $\phi 0 \mathrm{~mm}$ fixture. In contrast, no clear reduction of the electric voltage is seen for the $\phi 5 \mathrm{~mm}$ fixture after decrement of the voltage in the beginning of the cyclic loading. It should be pointed out that such energy loss could be attributed to the entropy generation. Namely, the entropy generation can be directly assessed with the failure of the PZT ceramics [12,13]. Figure 15 illustrates schematically the electric-power generation and failure characteristics for the PZT ceramic during the cyclic loading for $\phi 0$ and $\phi 5-\phi 20 \mathrm{~mm}$ fixtures, in which the mechanisms of electric generation and the reduction of the electric voltage for all loading fixtures are indicated.

at $100 \mathrm{~N}$

(a) Loading fixture $\phi 0 \mathrm{~mm}$

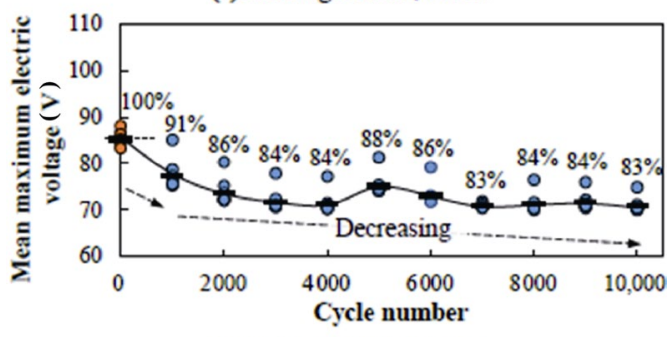

(b) Loading fixture $\phi 5 \mathrm{~mm}$

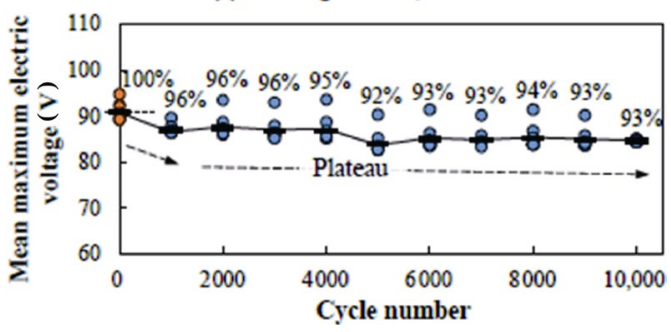

Fig. 14 Maximum voltage generated by PZT ceramic as a function of cycle number for (a) $\phi 0$ and (b) $\phi 5 \mathrm{~mm}$ loading fixtures at load of $100 \mathrm{~N}$. 


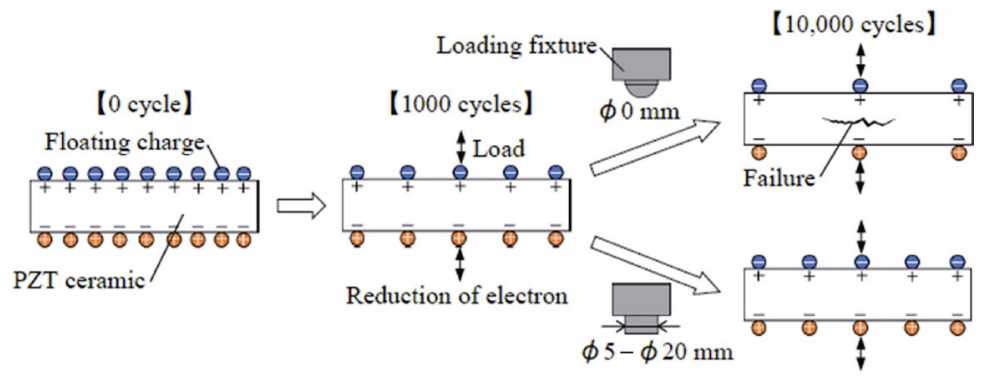

Fig. 15 Schematic diagram showing mechanism of electric-power generation and failure of PZT ceramic when cyclic loading is applied by $\phi 0 \mathrm{~mm}$ loading fixture.

\section{Conclusions}

Electric-power generation in piezoelectric PZT ceramic plates is investigated with the films subjected to mechanical loading by various loading fixtures with different contact areas. The main results are as follows:

(1) The generated voltage increases nonlinearly with decreasing contact area because of the increase in strain. A high voltage is produced for cyclic loading with a small contact area. The $\phi 0$ and $\phi 5 \mathrm{~mm}$ loading fixtures produce a similar high voltage, which is due to localized failure in the PZT ceramic caused by the $\phi 0$ $\mathrm{mm}$ fixture and the concomitant reduction in voltage.

(2) The strain characteristics (e.g., deflection, strain, and total equivalent strain) of PZT ceramic plate can be used to evaluate the voltage value. The generated voltage correlates strongly with deflection value, whereas the tensile and compressive strains affect the opposite electric generation voltage. Tensile strain is generated on the outer side of the PZT ceramic (near the edge of the testing stand), which leads to the generation of a negative voltage.

(3) The voltage generated during cyclic loading with the $\phi 0 \mathrm{~mm}$ fixture decreases with increasing cycle number because of material failure arising from the high stress concentration imposed by this fixture. In contrast, the voltage remains essentially constant when using a loading fixture greater than $\phi 5 \mathrm{~mm}$ in size. Considering the lack of material damage and the generation of high voltage, the $\phi 5 \mathrm{~mm}$ loading fixture is deemed optimal for an energy-generation system based on PZT ceramic plate. The area contact $(\phi 5 \mathrm{~mm})$ with the area ratio of 0.04 $(\phi 5 \mathrm{~mm} / \phi 25 \mathrm{~mm})$ is appropriated condition to obtain the high efficiency of the electric generation.

\section{References}

[1] Priya S. Advances in energy harvesting using low profile piezoelectric transducers. J Electroceram 2007, 19: 167-184.

[2] Sodano HA, Park G, Inman DJ. Estimation of electric charge output for piezoelectric energy harvesting. Strain 2004, 40: 49-58.

[3] Cook-Chennault KA, Thambi N, Sastry AM. Powering MEMS portable devices - a review of non-regenerative and regenerative power supply systems with special emphasis on piezoelectric energy harvesting systems. Smart Mater Struct 2008, 17: 043001.

[4] Sodano HA, Inman DJ, Park G. Comparison of piezoelectric energy harvesting devices for recharging batteries. $J$ Intell Mater Syst Struct 2005, 16: 799-807.

[5] Tadesse Y, Zhang SJ, Priya S. Multimodal energy harvesting system: piezoelectric and electromagnetic. J Intell Mater Syst Struct 2009, 20: 625-632.

[6] Shu YC, Lien IC. Efficiency of energy conversion for a piezoelectric power harvesting system. J Micromech Microeng 2006, 16: 2429-2438.

[7] Okayasu M, Sato D, Sato Y, et al. A study of the effects of vibration on the electric power generation properties of lead zirconate titanate piezoelectric ceramic. Ceram Int 2012, 38: 4445-4451.

[8] Okayasu M, Watanabe K. The electric power generation characteristics of a lead zirconate titanate piezoelectric ceramic under various cyclic loading conditions. Ceram Int 2015, 41: 15097-15102.

[9] Partridge J, Abouelamaimen DI. The role of supercapacitors in regenerative braking systems. Energies 2019, 12: 2683.

[10] Okayasu M, Watanabe K. A study of the electric power generation properties of a lead zirconate titanate piezoelectric ceramic. Ceram Int 2016, 42: 14049-14060.

[11] Okayasu M, Yamasaki T. Effects of $90^{\circ}$ domain switching on electric generation properties of PZT ceramic. Ceram Int 2017, 43: 3590-3600.

[12] Cuadras A, Romero R, Ovejas VJ. Entropy characterisation of overstressed capacitors for lifetime prediction. J Power Sources 2016, 336: 272-278.

[13] Hsiao CC, Liang BH. The generated entropy monitored by pyroelectric sensors. Sensors 2018, 18: 3320.

Open Access This article is licensed under a Creative Commons Attribution 4.0 International License, which permits use, sharing, adaptation, distribution and reproduction in any medium or format, 
as long as you give appropriate credit to the original author(s) and the source, provide a link to the Creative Commons licence, and indicate if changes were made.

The images or other third party material in this article are included in the article's Creative Commons licence, unless indicated otherwise in a credit line to the material. If material is not included in the article's Creative Commons licence and your intended use is not permitted by statutory regulation or exceeds the permitted use, you will need to obtain permission directly from the copyright holder.

To view a copy of this licence, visit http://creativecommons. org/licenses/by/4.0/. 